\title{
Junior College Library Statistics
}

Qince this is the deut of Junior Col$\checkmark$ lege Library Statistics to COLlege AND RESEARCH LIBRARIES, it must be stated that members of the Junior College Section are most grateful and appreciative for this printing.

In 1952-53, the first attempt was made to compile such figures on a national basis. Fifty schools responded before the deadline. The results were included in the "Junior College Libraries Section Newsletter" of November, 1953.

This year, when the call went out for statistics, the number of schools responding was doubled. Many librarians expressed their pleasure with ACRL for its promised support. The questionnaire employed was a shorter form than last year's and stressed points of special interest to our organization.

The committee members who worked on the questionnaire form included: $M$ iss Daisy L. Anderson of Mars Hill College, Miss Ruth Gray of San Angelo College, Miss Nellie M. Homes of Cottey College, Mrs. Claryse D. Myers of Tennessee Wesleyan College, Mrs. Lola Rivers Thompson of Tarleton State College, and your Chairman of Statistics for 1953-54, Mrs. Thelma Vogt Taylor of Los Angeles Harbor Junior College.

Statistics were received from I Io schools. Giffin College has been omitted since it uses the facilities of the public library. Some gaps exist because no figures were submitted in these columns. It is interesting to note that information on salaries is much more complete than last year. Many schools are allowed to report existing schedules but are hesitant about revealing personal data.

In the 109 schools included in these statistics, the head librarian has the status of administrator in 5 junior colleges, that of coordinator in 2, department chairman in $I I$. In the majority of schools, however, the librarian is still ranked as a teacher.

Since there is such a wide variation in schools and their resources, the statistics given are descriptive only. No attempt is made to evaluate or include medians. It is hoped that the results will be of value and interest to our members.-Thelma $V$. Taylor, Chairman, Statistics Committee, Junior College Section.
This year, for the first time, collEge AND RESEARCH LIBRARIES is happy to include the Junior College Library Statistics. Although the time was short, the Statistics Committee of the Junior College Section swung into high gear and got their statistics to us promptly. Since this is their first appearance, and the result of some hurried action on the part of all of us, we ask your patience and toleration. We tried to go over the statistics carefully in an effort to bring them into line with our "College and University Library Statistics," but some variations had to remain. Since these statistics were not originally collected for inclusion in COLLEGE AND RESEARCH LIBRARIES, some production problems presented themselves and led to minor revision of the report first submitted by the Junior College Committee.

The following headings in the original report have been omitted here because data were either incomplete or did not seem significant:

Newspapers (number in library)

Periodicals (number in library)

Two-week and reserve circulation-yearly circulation figures are included and it is felt that these are the most significant.

Total hours weekly and salaries (clerical workers)-these figures were collected but were not included here since they are dependent on the local situation, and did not appear to be significant for this report. The number of clerical workers is equated to a full-time equivalent.

Hourly rate of student assistants-hours of student assistance is included and seems to be the more significant.

Library facilities-a footnote has been used to indicate those institutions which have separate library buildings. All other libraries are housed in a building used for other purposes.

These Junior College Statistics were compiled by the Statistics Committee of the Junior College Section under the chairmanship of Mrs. Thelma Vogt Taylor, librarian, Los Angeles $\mathrm{Harbor}$ Junior College. To her, and to Nellie Homes, chairman of the Junior College Section, go our sincere thanks for their cooperation in preparing the statistics for print in time for the January issue.-Cynthia Spigelman, Publications Officer. 


\section{Junior College Library Statistics, $1953-54$}

\section{- Enrollment —_}

College

1. Abraham Baldwin Agric. Coll., Ga. 2. Anderson College, S.C. . 4. Armstrong Coll of., Calif..
Day Night Board-

Day Night ing Hours Circula- Vol-

dents dents dents Wk. tion umes Book

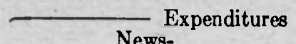

Nexpenditures

Personnel

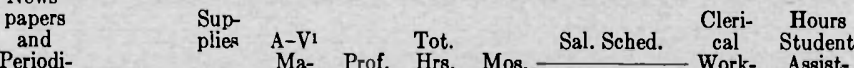

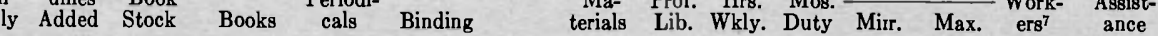

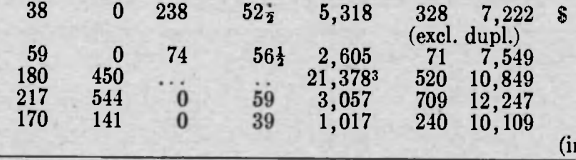

7. Austin Jr. Coll., Mi., Bacone Coll. Okla.
8. Bakersfield Coll.,

9. Becker Jr. Coll., Mass

11. Bluefield Coll., Va.

12. Brevard Coll., N.C.

14. Cazenovia Jr. Coll., N.Y.Y. $\begin{array}{ccccc}40 & 2,854 & 175 & 3,366 & 486 \\ 45 & 3,581 & 433 & & \\ 4 & & \end{array}$

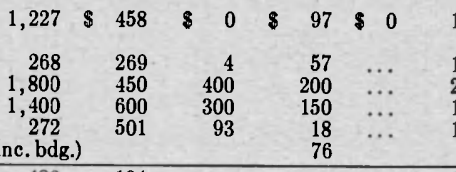
\begin{tabular}{llll}
50 & 3,000 & 1,100 & 6,800 \\
\hline & 3805 & 8,700
\end{tabular} $\begin{array}{llll}59 & 2,643 & 543 & 7,751\end{array}$

15. Chaffey Jr. Coll., Calif. ${ }^{2} \ldots \ldots \ldots \ldots \ldots \ldots . \quad 835 \quad 350 \quad \ldots$ $\begin{array}{llll}56 \frac{1}{2} & 4,307 & 379 & 15,753\end{array}$ $\begin{array}{rrrr}73 & 9,776 & 277 & 6,888 \\ 54 \frac{1}{2} & 17,741 & 1,162 & 14,974\end{array}$ $\begin{array}{llll}60 & 28,423 & 575 & 48,000\end{array}$ $\begin{array}{lrrr}401 & 9 & 7,7 & 17,700 \\ 80 & 10 & 3,700 & \$ 5,700 \\ 37 & 12 & 3,710 & 3,820 \\ 39 & 12 & 2,575 & \end{array}$

\begin{tabular}{rrrrrrrrrrrr}
194 & $\ldots$ & & $\ldots$ & 1 & 40 & 9 & 3,300 & 5,700 & $\frac{1}{3}$ & 0 & 6 \\
397 & 600 & $\ldots$ & $\mathbf{3 0 0}$ & 1 & $35-38$ & $9 \frac{1}{2}$ & 3,000 & $\ldots \ldots$ & 0 & 61 & 7 \\
250 & 275 & 375 & $\ldots$ & 1 & 35 & $9 \frac{1}{2}$ & $\ldots \ldots$ & $\ldots \ldots$ & 1 & 50 & 8 \\
675 & 0 & 75 & $\ldots$ & 1 & 40 & 9 & $\ldots \ldots$ & $\ldots \ldots$ & 0 & $\ldots$ & 9 \\
300 & 350 & 91 & 600 & 1 & 40 & 9 & $\ldots \ldots$ & 4,250 & $\frac{1}{4}$ & 30 & 10 \\
\hline 229 & 0 & 84 & & 1 & 44 & 9 & 2,700 & 4,050 & 0 & 42 & 11 \\
436 & 188 & 100 & 251 & 1 & $39 \frac{1}{2}$ & 9 & 3,200 & $\ldots \ldots$ & $\frac{3}{3}$ & 60 & 12 \\
112 & 183 & 333 & $\ldots$ & 1 & 40 & 10 & 3,900 & $\ldots \ldots$ & $\frac{1}{2}$ & 4 & 13 \\
479 & 126 & $\ldots \ldots$ & 244 & 1 & $37 \frac{1}{2}$ & 11 & 2,500 & 4,500 & 2 & 33 & 14
\end{tabular}

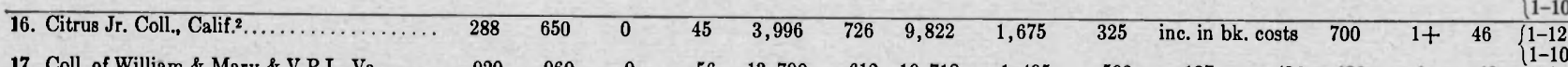

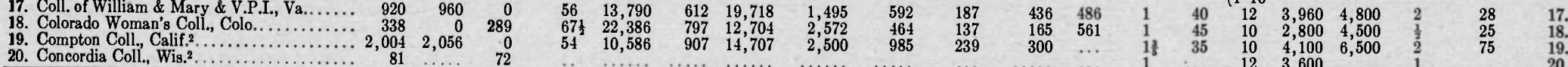

21. Connors State Ag. Coll., Okla

(0....... $1,531 \quad 2,057$

24. El Camino Coll., Calif. ${ }^{2}$.
25. Endicott Jr. Coll., Mass.

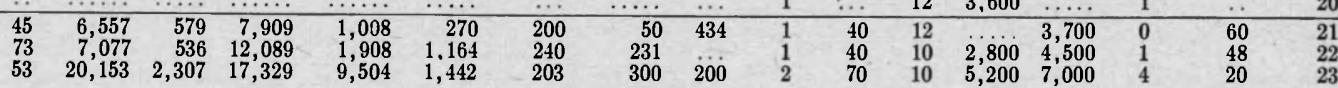

26. Everett Jr. Coll., Wash.

27. Forida Christian Coll., Fla.....................

29. Georgetown Visitation J.. Coll., Wash. D.C. gia Military Coll., Ga.

31. Georgia South western Coll., Ga.2 33. Hannibal-La Grange, M

34. Hartford Coll., Conn

36. Henry Ford Com. Coll., Mich.

37. Hesston Coll., Kan

Holton-Arms School \& Jr. Coll, Wash., D.C

40. Hutchingon $\mathrm{Jr}_{\mathrm{r}} \mathrm{Jr}_{\mathrm{i}}$ Coll.,

. Jackson Jr. Coll., Mich...

33. Jefferson City Jr. Coll., Mo

44. Johnstown Center, U. of Pitts., Pa.

. Joplin Jr. Coll., Mo.

6. Keystone Jr. Coll., Pa...

$\begin{array}{llll}223 & \ldots & \ldots & \\ 531 & 2,057 & \cdots\end{array}$

566

501
605
2,769 -12 pl

15.

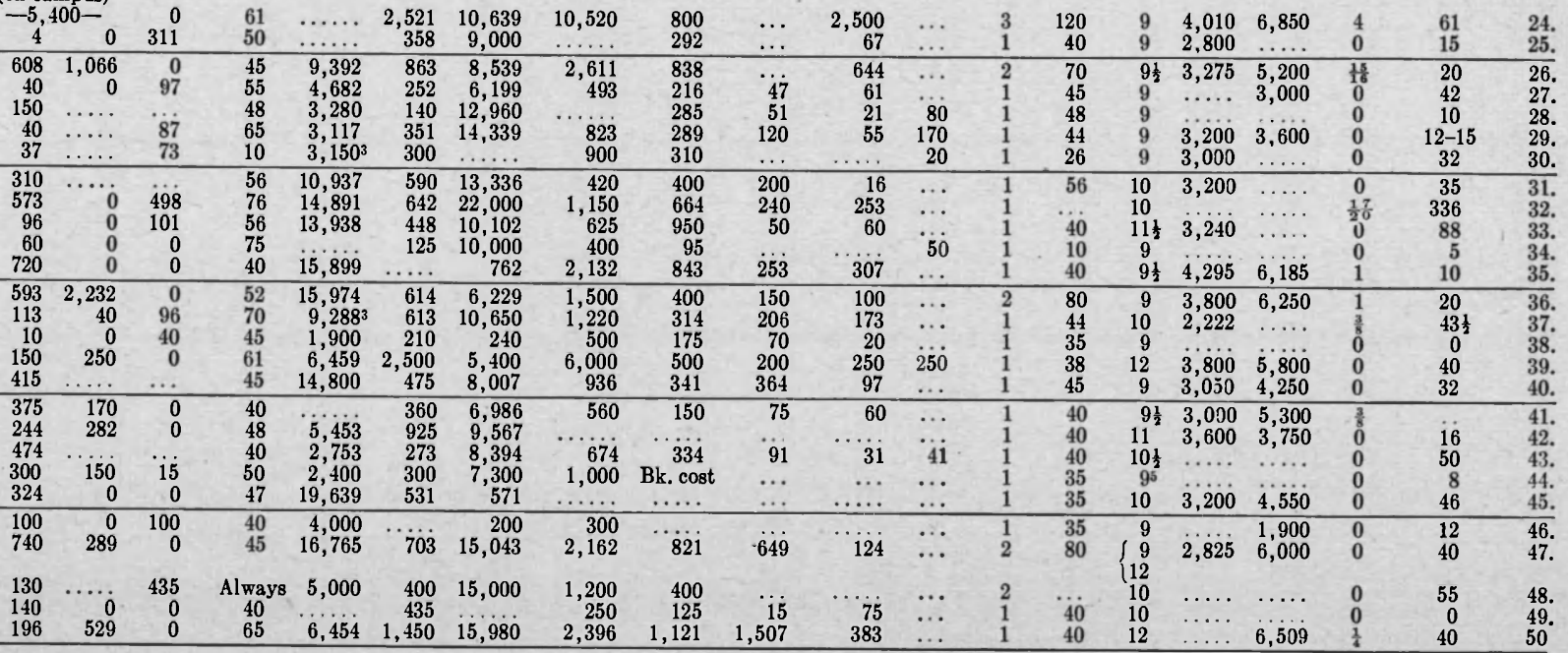


51. Lees-McRae Coll., N.C.
52. Lineoln Coll... Ill.

53. Long Beach City Coli., Cali

55. Loretto Jr. Coll., Ky. Kos Angeles City Coll., Calif.2.

57. Los Angeles Trade-Tech. Jr. Coll., Calif..... $3,000 \quad 4,000$ 99. Marion Institute,

60. Mars Hill Coll., N.C.2

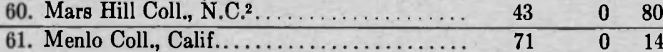

63. Mohawk Valley T. I., N.Y

65. Montgomery . Coll., N. Maj

66. Morton, Jr. Coll., Sterling, Ill......

67. Mt. Vernon Jr. Coll., Wash., D.C

69. Murtnomah Coll., Ore.... Dakota State School of Sci., N.D...

\begin{tabular}{rr}
110 & 0 \\
336 & 750 \\
316 & 530 \\
372 & 127 \\
\hline 372 & 169
\end{tabular}

70. Northern Montana Coll., Mont.

71. Northern Oklahoma Jr. Coll., Okla.......

73. Olympic Coll Was

74. Orange Coast Coll., Calif.
75. Palm Beach Jr. Coll., Fla.

Coll., Calif. $2,6 \ldots \ldots \ldots \ldots .4,765 \quad 1,950 \quad 0$

77. Penn State Coll. Ogontz Center, $\mathrm{Pa}$

$\begin{array}{rrr}405 & \cdots & 60 \\ 24 & 0 & 718 \\ 32 & 53 & 260 \\ 85 & 70 & 50\end{array}$

$\begin{array}{llll}40 & 2,535 & 764 & \\ 37 \frac{1}{2} & 7,500 & 407 & 8,424 \\ 30 & 5,516 & 316 & 5,507 \\ 50 & 4,450 & 200 & 6,800\end{array}$

1,000 Bk. Cost
952

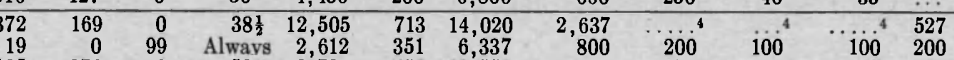

$\begin{array}{rrrrrrrrr}105 & 376 & 0 & 52 & 2,784 & 450 & 10,556 & 850 & 375 \\ 110 & \ldots \ldots & 490 & 49 & 8,526 & 601 & 14,500 & 2,443 & 1,362\end{array}$

79. Pineland Jr. Coll.., N.C. N. . .

81. Riverside Coll. Coll., N.C.

\begin{tabular}{rrrrrrrr}
49 & 8,526 & 601 & 14,500 & 2,443 & 1,362 & 0 & 452 \\
58 & 5,860 & 683 & 22,761 & 1,642 & 394 & 249 & 112 \\
\hline 48 & $\dddot{2} \ldots$ & 400 & 11,800 & $7 \ldots 0$ & -100 & $\cdots$ & $\cdots$ \\
60 & 4,609 & 461 & 8,854 & 1,300 & 400 & 0 &
\end{tabular}

$\begin{array}{rrr}4,046 & 412 & 6,486\end{array}$

$\begin{array}{rrrr}45 & 6,223 & 1,623 & \ldots, 337\end{array}$

$\begin{array}{rrrrr} & 400 & 100 & 200 \\ \mathbf{5}, 500 & 1,159 & 57 & 40 & 00\end{array}$

$\begin{array}{rrrrrrrrrr}53^{\frac{3}{4}} & 64,528 & 2,294 & 50,354 & 9,153 & 1,660 & 2,000 & 3,000 & 300 & 6\end{array}$

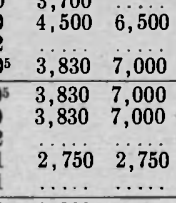

40
100

82. Rochester Jr. Coll., Minn.............. 235 1,755 $\quad 00$

84. St. Mary's School \& J.C., N.C..............

86. St. Petersburg J.C., Fla

87. San Angelo Coll, Texas.

89. San Antonio Coll., Texas.......... Cali

90. San Diego Jr. Coll., Calif.

91. Santa Ana Coll., Calif. Santa Maria Jr. Coll., Calif.

93. Santa Monica City Coll., Calif. 2

95. Sierra Coll., Calif.. Couthwest Baptist Coll., Mi.

$\begin{array}{lrrr}40 & 2,621 & 203 & 9,000\end{array}$

3,831
624
766 \begin{tabular}{rrr}
$55+\quad 2,718$ & 333 & 10,000 \\
\hline
\end{tabular}

\begin{tabular}{rrrrrr}
276 & $\dddot{50}$ & $\mathbf{5 0}$ & & 1 & 4 \\
629 & 445 & 1,665 & 140 & 6 & 24 \\
179 & & 67 & $\ldots$ & 1 & 3 \\
249 & $\dddot{5} 2$ & 25 & 25 & 1 & 40 \\
\hline
\end{tabular}

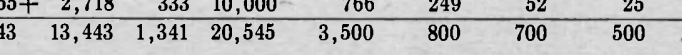

96. Stockton Coll., Calif. $8 . \ldots \ldots \ldots \ldots \ldots \ldots \ldots, 3,137$

97. Sue Bennett Coll., Ky............ Tarleton State Coll., Texa.

99. Tennessee Wesleyan Coll., Tenn

100. Ursuline Coll. of Paola, Kan

101. Vallejo Coll., Calif.2,6.

103. Victoria Coll., Tex

104. Virginia Intermont

106. Warren Wilson Coll., N.C. 2

$\begin{array}{rrr}235 & 1,755 & 0 \\ 25 & \cdots \cdots & 40 \\ 31 & 24 & 258 \\ 580 & 0 & 31 \\ 500 & 175 & 125 \\ 720 & 1,950 & 0 \\ 300 & 2,000 & 0 \\ 643 & 633 & 0 \\ 513 & 2,027 & 0 \\ 142 & 2 & 0 \\ 210 & 4,443 & 0 \\ 407 & 721 & 0 \\ 420 & 0 & 296\end{array}$

$\begin{array}{llll}\mathbf{4 5} & 4,223 & 208 & 8,938 \\ \mathbf{5 4} & 2,697 & 230 & 6,472\end{array}$

900
549
932

$\begin{array}{lrrrr}66 & 1,005 & 557 & 12,662 & 1,65\end{array}$

$\begin{array}{rrrr}52 & 7,656 & 371 & 9,400 \\ 69 & 12,692 & 1,152 & 12,355\end{array}$

$\begin{array}{rrrr}65 & 12,692 & 1,152 & 12,355 \\ 55 & 12,820 & 1,027 & 35,228 \\ 65 & 9,725 & 1,957 & 2,088\end{array}$

107. Weber Coll., Uta

$\begin{array}{rrr}37 & 0 & 0\end{array}$

$\begin{array}{llll}39,000 & 721 & 10,001\end{array}$ $\begin{array}{llll}48 & \mathbf{7}, 320 & 1,046 & 14,875 \\ \mathbf{5 4} & \mathbf{5}, 466 & 1,000 & 12,500\end{array}$

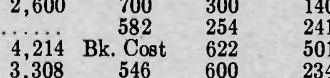

108. West Contra Costa J.C., Calif \begin{tabular}{rrr}
80 & $\cdots \cdots$ & 30 \\
144 & $\cdots$ & 483 \\
29 & 0 & 148 \\
\hline 2 & &
\end{tabular}

$\begin{array}{lllll}58 \frac{1}{4} & 62,720 & 4,065 & 13,614 & 70,000\end{array}$

$150 \quad \ldots$

$\begin{array}{crrrr}\begin{array}{crcc}700 \\ \text { for all }\end{array} & & \ldots & 1 & 3 \\ 32 & 23 & \cdots & 2 & 5 \\ 168 & 131 & \ldots & 1 & 3 \\ 165 & 127 & 380 & 1 & 27 \\ 70 & 40 & \ldots & 1 & 4 \\ 304 & 158 & \cdots & 1 & 4 \\ 289 & 680 & 67 & 2 & 8 \\ 400 & 400 & 150 & 3 & 10 \\ 62 & 195 & \ldots & 2 & 80\end{array}$

$\begin{array}{rrrrrrc}38 & 4,339 & \ldots & 12,300 & 251 & 288 & 60 \\ 66 & 68,448 & 790 & 36,825 & 3,116 & 770 & \text { Bk. Cost } \\ 48 & 9,822 & 552 & 18,365 & 796 & 441 & 212 \\ 60 & 5,166 & 526 & 8,959 & 473 & 181 & 35\end{array}$

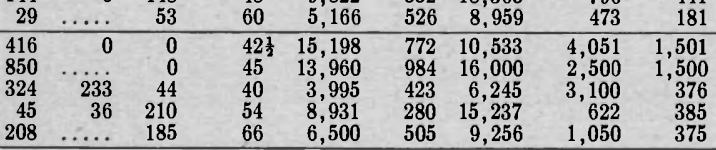
$\begin{array}{lllllll} & 2 . .6 & 185 & 66 & \mathbf{6 , 5 0 0} & \mathbf{5 0 5} & 9,25 \\ \end{array}$ \begin{tabular}{rrr}
375 & 214 & 93 \\
358 & 11 \\
\hline
\end{tabular} $\begin{array}{rrr}9,911 & 1,222 & 14,670 \\ 24,866 & 1,100 & 26,000\end{array}$

$\begin{array}{rrrr}508 & 227 & 65 & 88\end{array}$

$\begin{array}{lllllllllllllllllll}1 \text { If } & 54 \frac{1}{2} & 8,627 & 398 & 14,387 & 726 & 274 & 164 & 211 & \ldots & 1 & 40 & 11 & \ldots & 2,400 & 0\end{array}$

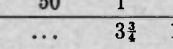
$12,3,115$ $\begin{array}{lll}60 & 6,390 & 0 \\ 3 & \ldots \ldots & 3 \\ 3,316 & 1 \\ 4,600 & ? \\ 50 & 4,450 & 5 \\ 00 & 4,800 & 0 \\ 00 & 5,800 & 0 \\ 50 & 6,350 & 1 \\ 00 & 6,500 & 1 \\ 00 & 6,525 & 0 \\ 00 & 6,000 & 1 \\ 24 & 7,263 & 2 \\ 50 & 6,450 & 0 \\ 00 & \ldots \ldots & 0 \\ 15 & 7,430 & 2\end{array}$ \begin{tabular}{cc}
20 & 82 \\
0 & 83 \\
271 & 84 \\
21 & 85 \\
\hline 0 & 86 \\
56 & 87 \\
20 & 88 \\
40 & 89 \\
23 & 90 \\
14 & 91 \\
$7 \frac{1}{2}$ & 92 \\
25 & 93 \\
28 & 94 \\
63 & 95 \\
\hline
\end{tabular} $\begin{array}{rrrrrcrrrr} & & 1 & 38 & 9 & 2,400 & 0 & 20 & 97 . \\ 1 & \cdots & 3 & 120 & 12 & 3,500 & 5,200 & 2 & 128 & 98 . \\ & 1 & 50 & 12 & \ldots \ldots & 3,100 & \frac{1}{3} & 44 & 99 .\end{array}$

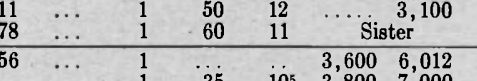

8

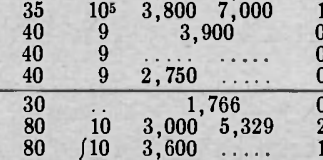
$\cdot$ $\begin{array}{ll}75 & 102 \\ 30 & 103 \\ 53 & 104 \\ 66 & 105\end{array}$ $\begin{array}{ll}45 & 106 \\ 56 & 107 \\ 60 & 108\end{array}$ . 